This document is confidential and is proprietary to the American Chemical Society and its authors. Do not copy or disclose without written permission. If you have received this item in error, notify the sender and delete all copies.

\title{
Staggered fibrils and damageable interfaces lead concurrently and independently to hysteretic energy absorption and inhomogeneous strain fields in cyclically loaded antler bone
}

\begin{tabular}{|r|l|}
\hline Journal: & ACS Biomaterials Science \& Engineering \\
\hline Manuscript ID & ab-2016-00637q.R1 \\
\hline Danuscript Type: & Article \\
\hline Complete List of Authors: & $\begin{array}{l}\text { De Falco, Paolino; Queen Mary University of London, School of Engineering } \\
\text { and Materials Science } \\
\text { Barbieri, Ettore; Queen Mary University of London, School of Engineering } \\
\text { and Materials Science } \\
\text { Pugno, Nicola; Università di Trento, Department of Civil, Environmental } \\
\text { and Mechanical Engineering; Queen Mary, University of London, School of } \\
\text { Engineering and Materials Science; Fondazione Bruno Kessler, Center for } \\
\text { Materials and Microsystems } \\
\text { Gupta, Himadri; Queen Mary University of London, School of Engineering } \\
\text { and Materials Science }\end{array}$ \\
\hline
\end{tabular}




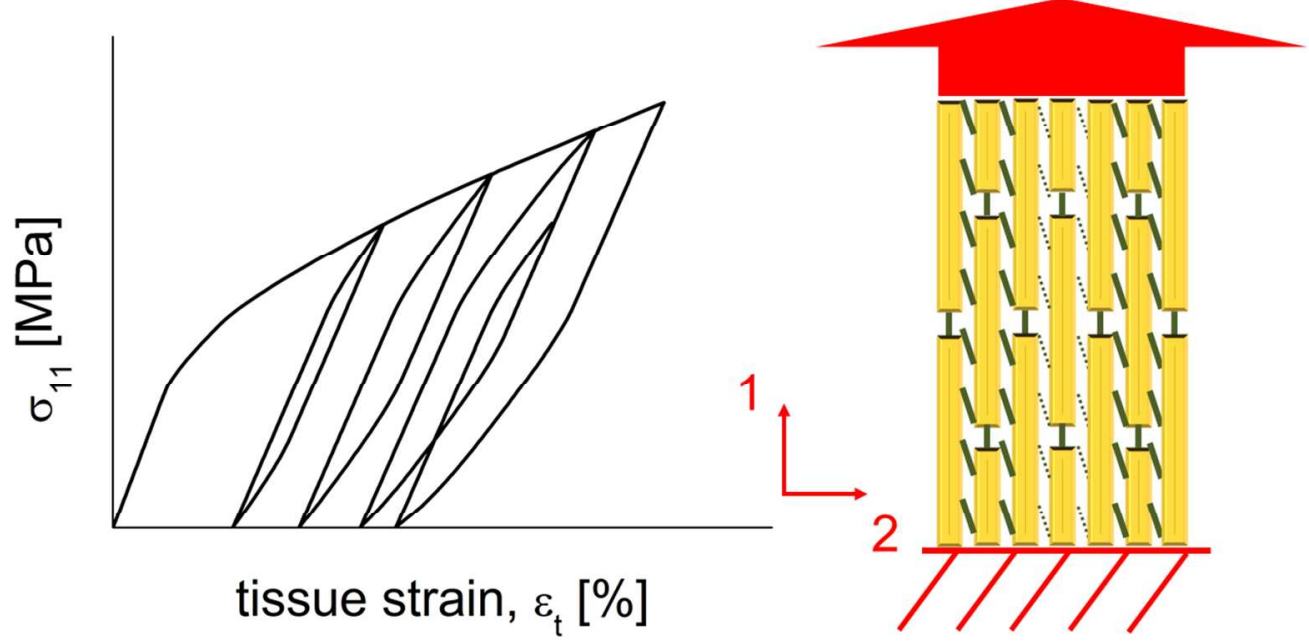

Hysteresis at the fibrillar level in the mineralized fibrils of antler bone (left) is enabled via the staggered architecture and interfaces which can fail above critical loads (right)

$334 \times 165 \mathrm{~mm}(96 \times 96 \mathrm{DPI})$ 


\title{
Staggered fibrils and damageable interfaces lead concurrently and
}

\section{strain fields in cyclically loaded antler bone}

\author{
P. De Falco ${ }^{a}$, E. Barbieri ${ }^{a}$, N. Pugno ${ }^{a, b, c}$ and H. S. Gupta ${ }^{a^{*}}$ \\ ${ }^{a}$ Queen Mary University of London, School of Engineering and Material Science, London, E1 4NS, UK \\ ${ }^{b}$ Laboratory of Bio-Inspired and Graphene Nanomechanics, Department of Civil, Environmental and \\ Mechanical Engineering, University of Trento, Trento, Italy \\ ${ }^{c}$ Center for Materials and Microsystems, Fondazione Bruno Kessler, Povo (Trento), Italy \\ *Corresponding author: Himadri S. Gupta (h.gupta@qmul.ac.uk)
}

\section{Keywords}

Antler bone, interface, nanoscale, cohesive behaviors, heterogeneity, hysteresis

\section{Abstract}

The high toughness and work to fracture of hierarchical composites, like antler bone, involve structural mechanisms at the molecular, nano- and micro scales, which are not completely explored. A key characteristic of the high energy absorption of such materials is the large hysteresis during cyclic loading, but its origin remains unknown. In situ synchrotron X-ray diffraction tests during tensile loading of antler bone showed heterogeneous fibrillar deformation and hysteresis. To explain the origin of these mechanisms from the nanostructure of antler bone, here we develop a class of finite-element fibril models whose predictions are compared to experimental data across a range of potential composite architectures. We demonstrate that the key structural motif enabling a match to experimental data is an axially staggered arrangement of stiff mineralized collagen fibrils coupled with weak, damageable interfibrillar interfaces.

\section{INTRODUCTION}

Natural structural materials exhibit mechanical properties through complex hierarchical architectures and load-absorbing mechanisms. These architectures evolved naturally from basic building blocks thanks to a 'self-organization' strategy during growth ${ }^{1}$. In fact, biological structures adapt, change function during growth, renew their material and build hierarchies $^{2}$. The macroscopic behaviour of these materials depends on the interaction 
29 between structural properties at different scales ${ }^{3}$. Bio-composites, such as bone, shells 30 and nacre, represent an excellent example of how the design at lower hierarchical scales 31 confers higher mechanical properties than the single constituents ${ }^{4}$. Although the stiffness 32 of these biocomposites is comparable to that of the basic constituent at the nanoscale, 33 their toughness results hugely increased. For instance, in bone and shell, the toughness of 34 the mineral constituents is $\ll 1 \mathrm{MPa}^{*} \mathrm{~m}^{1 / 2}$ while the toughness of their macrostructure 35 varies, respectively, in a range of $2-7 \mathrm{MPa}^{*} \mathrm{~m}^{1 / 2}$ and $3-7 \mathrm{MPa}^{*} \mathrm{~m}^{1 / 2}$.

Bone, as shown in Figure 1, at the nanometre scale length is a composite of stiff inorganic hydroxyapatite platelets interleaved with a softer organic matrix, made principally of type I tropocollagen proteins ${ }^{5}$. This sub-structure, together with an intrafibrillar phase of noncollageneous proteins and mineral, forms mineralized fibrils that are arranged into 41 aggregate structures at higher levels and larger length scales, such as fibril arrays and 42 lamellae ${ }^{1}$. The structural aspects of this architecture served as inspiration for bioinspired 43 materials that replicate the nanometre scale fibril-matrix ${ }^{6-10}$ and intrafibrillar ${ }^{11}$ structure, or 44 at micrometre scales ${ }^{12,13}$. Nonetheless, the mechanical interactions between the 45 constituent units and the higher length scales remain a matter of active research. In particular, previous studies focused on how the hierarchical architecture brings functionally desirable properties such as high toughness ${ }^{14}$, energy absorption and fatigue resistance ${ }^{15}$.

At the range of $1-100 \mu \mathrm{m}$, accepted and validated toughness mechanisms are crack 50 deflection and bridging ${ }^{16}$, and constrained microcracking ${ }^{17}$. The nanoscale structure is 51 believed to be of fundamental importance for bone toughness. However, it is both 52 challenging to investigate experimentally ${ }^{18}$ as well as to explain the reasons of its 53 mechanical properties at this scale with a model. Works to date mainly focused on either 54 deformation beyond the yield point under uniaxial or localized loading ${ }^{18-20}$ or on post-hoc 
55 interpretation of electron microscopic images of loaded and fractured bone ${ }^{21-22}$. These

56 experimental studies led to hypothesise different toughness mechanisms ${ }^{23}$. Examples 57 include intrafibrillar plasticity ${ }^{20}$, sacrificial bonds within noncollageneous proteins ${ }^{22}$, friction 58 between collagen and mineral ${ }^{24}$, fibrillar sliding of mineralized collagen fibrils ${ }^{25}$, interfibrillar 59 sliding of collagen fiber arrays ${ }^{26}$ and microcracking ${ }^{27}$. At these small length scales 60 relatively less clear evidence exists on the response to cyclic loading, although recent 61 experimental work has begun to shed light on this question. For example, Schwiedrzik et 62 al. ${ }^{28}$ focused on compression and cyclic micro-pillar tests on lamellar bone and measured 63 axial and transverse apparent moduli and compressive strengths.

64 Bone is physiologically subjected to external periodic loading that can lead to fatigue failure, and high rate impact that instead can lead to fracture. It is then of considerable

67 interest to understand how the nanostructure behaves under these loading conditions. 68 Unfortunately, experimental information on the structural changes at the fibrillar and 69 interfibrillar level in these loading modes is relatively scarce. Concurrently, the link 70 between the types of fibrillar architecture and the developed cyclic inelastic response is 71 also not very clear. In this regard, a recurrent generic motif in the architecture of hard 72 biological composites is a staggered arrangement of fibrils (Figure $\mathbf{2 b}$ ) $^{29}$. This particular 73 arrangement plays a key role in energy dissipation through sliding ${ }^{30-31}$ and in enhancing 74 the structural elastic properties ${ }^{32-33}$. Gupta et al. ${ }^{20}$ identified elastoplastic behaviour for the 75 individual mineralized fibril under the assumption of staggered configuration of mineral 76 platelets and collagen molecules inside the fibril.

78 The role of such a staggered configuration in cyclic loading and energy absorption is 79 unexplored at the nanoscale. Recent in situ synchrotron SAXD/WAXD mechanical 80 loading/unloading tests on antler bone ${ }^{20}$ show hysteresis in stress-strain curves at both the 
81 macroscopic and the fibrillar level. These results also highlight the presence of two groups 82 of fibrils: plastically deforming fibrils, which exhibit larger deformation (which will be 83 denoted type $\mathrm{A}$ in what follows), and elastically deforming fibrils (denoted type B), whose 84 deformation remains at or under the strain at the material yield point. It is clear that these 85 structural mechanisms may be related, and that the fibrillar hysteresis is an important 86 component of the high work to fracture of antler, but its structural origins are far less 87 understood. In situ experimental probes of the type described above need to be combined 88 with ultrastructural modelling at the scale of $1-100 \mathrm{~nm}$, in order to develop a deeper 89 understanding of the relevant mechanisms.

90

91 In this paper, we present a set of finite element simulations of the mineralized fibrils in 92 antler bone under cyclic loading whose results, when matched to experiment, give an 93 understanding of the causes of the fibrillar hysteresis. We will show that the combination of 94 a damageable interface and staggered fibrillar arrangement turns out to be capable of 95 explaining the experimentally observed hysteretic loops in loading/unloading curves. In 96 addition, a clear explanation of the biphasic fibrillar deformation mechanisms, in terms of 97 the dependence on interfacial strength and architecture, is here reported. These results 98 provide new insights of toughening mechanisms at the nanoscale in antler bone. 
url $9^{\cdot} \varepsilon \sim$

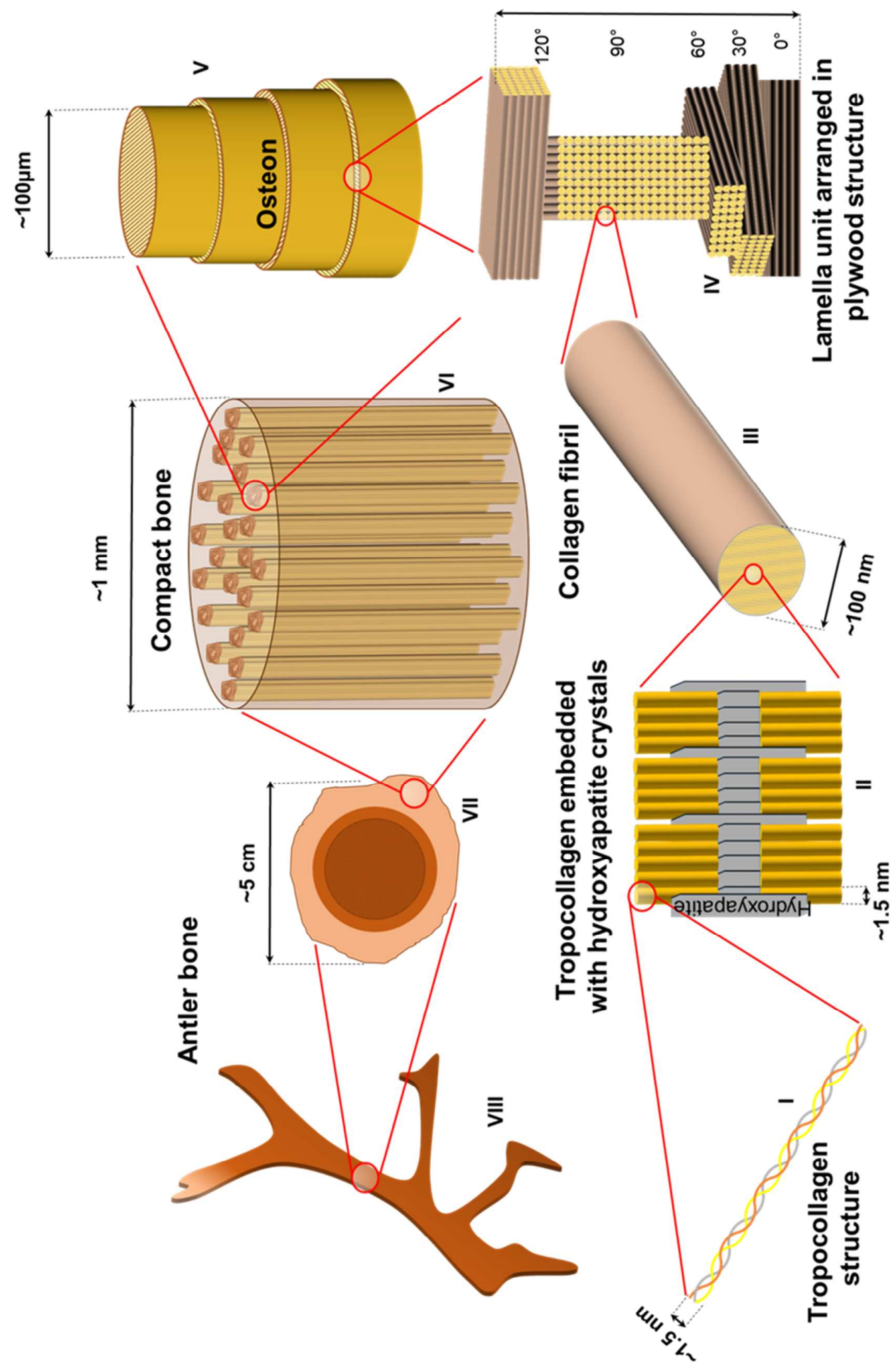


100 Figure 1 - Hierarchical structure in antler bone: The collagen fibrils (II) are made of 101 tropocollagen molecule (I) and hydroxyapatite mineral. At the following hierarchical level, 102 these fibrils are wrapped in a protein-based matrix (III) forming a plywood structure: the 103 lamella unit (IV). This group is repeated in the osteon (V) which is part of the compact 104 bone (VI) and therefore of the antler bone structure (VII-VIII).

105

106

\section{METHODS}

107

108

\subsection{Experimental method}

109 The preparation description of antler bone specimens and the in situ mechanical tests with synchrotron small angle X-ray diffraction (SAXD) are described in detail in a previous paper $^{20}$, and summarized here briefly. The samples were taken from the antler cortical shell of a red deer (Cervus elaphus) near the antler-pedicle junction and tested with SAXD measurements, combined with cyclic loading. While the details are available in our previous papers ${ }^{34}$, they will be summarized here for completeness. Figure 2 a shows a highly simplified schematic for tensile cyclic tests on hydrated antler bone specimens

116 combined with time-resolved synchrotron SAXD measurements, where a synchrotron X117 ray beam impinges on the specimen, leading to a sequence of SAXD patterns acquired as 118 the sample is deformed.

119

120 Meridional peaks are visible in the SAXD pattern, due to the periodic electron density 121 profile arising from the axial D-stagger of the tropocollagen molecules inside the fibril 122 (D 65-67 nm for vertebrate collagenous tissues). Percentage shifts in these peak positions 123 are therefore measures of fibril strain, as reported previously for bone (e.g. Gupta et al. ${ }^{35}$, 124 Dong et al. ${ }^{36}$, Zimmermann et al. ${ }^{37}$,among others). The $3^{\text {rd }}$ order meridional peak was used 
125 for determination of mean fibril strain, via the relation $D=6 \pi / q_{03}$ where $q_{03}$ was the peak 126 position, in reciprocal space, of the meridional peak. Further, the peak width $w_{\mathrm{q}}$ was also 127 determined, which (as reported in Krauss et al. ${ }^{34}$ and Gupta et al. ${ }^{20}$ ) provides a measure of 128 the heterogeneity of fibrillar deformation: a narrow $w_{\mathrm{q}}$ corresponds to a uniform fibrillar 129 deformation with all fibrils in the scattering volume deforming similarly, while an increasing $130 w_{\mathrm{q}}$ corresponds to an increasing heterogeneity, or dispersion, in the fibril strain distribution. 131 As the details are presented in Gupta et al. ${ }^{20}$, we note only that by tracking the stress132 induced increase in mean fibril strain, together with the increase in $w_{\mathrm{q}}$, a biphasic fibrillar 133 deformation was observed, and will serve as part of the comparison of our presented 134 model to experiment.

\subsection{Numerical method and implementation of the model}

136 Parametric finite elements simulations were performed to test a seven-layer staggered 137 fibrillar system using Abaqus (Abaqus 6.14-1, Dassault Systemes). The two-dimensional 138 model is made of 2800 CPS4R finite elements (CPS4R corresponds to 4-node, reduced 139 integration with hourglass control). The plates, measuring $10 \mu \mathrm{m} \times 0.2 \mu \mathrm{m}$, represent the 140 fibrils (with $200 \mathrm{~nm}$ or $0.2 \mu$ radius $^{2}$ ) in bone and are connected through cohesive laws, 141 which are shown in detail in Figure $\mathbf{2 b}^{38}$. Interfaces link both the lateral sides of fibrils 142 ('mode I interface') and their bottom-up sides ('mode II interfaces') (see Figure 3c for 143 interfaces definition). The model (Figure 2b) assumes initially linear elastic behaviour 144 (Equation 1, next page) followed by the initiation and evolution of damage. In Equation 1, 145 the traction stress vector consists, as our models are two-dimensional, of two components $146 t_{n}$ and $t_{s}$, which are, respectively, the normal and shear tractions, and $\delta_{n}$ and $\delta_{s}$ which 147 represent the relative displacements between the nodes on the adjacent surfaces of two 148 different fibrils. We decided to use the simplest traction-separation law, where normal $\left(\mathrm{K}_{\mathrm{nn}}\right)$ 149 and tangential $\left(\mathrm{K}_{\mathrm{ss}}\right)$ stiffness are not coupled $\left(\mathrm{K}_{\mathrm{ns}}\right.$ and $\mathrm{K}_{\mathrm{sn}}$ are null in Equation 1$)$. 


$$
t=\left[\begin{array}{l}
t_{n} \\
t_{s}
\end{array}\right]=\left[\begin{array}{cc}
K_{n n} & 0 \\
0 & K_{s s}
\end{array}\right]\left[\begin{array}{l}
\delta_{n} \\
\delta_{s}
\end{array}\right]
$$

150

151 As peak traction values for the mode I and mode II undamageable interfaces, we used 152 generic values such as $t_{n}^{0}=t_{s}^{0}=80 \mathrm{MPa}$. These values are never achieved among the 153 finite elements adjacent to the interfaces and do not affect the results. For mode II 154 damageable interfaces we adopted the values $t_{n}{ }^{0}=80 \mathrm{MPa}$ and $t_{s}{ }^{0}=0.8 \mathrm{MPa} ; \mathrm{t}_{n}{ }^{0}$ is an 155 arbitrary high value, never reached upon the structure, while $t_{s}{ }^{0}$ is the shear stress 156 occurring when at least one point in the structure reaches yielding. We followed the 157 hypothesis ${ }^{20}$ that heterogeneity, due to progressive mode II interface damage, starts 158 occurring in correspondence of the yielding point. We imposed this condition by choosing 159 as shear traction peak value, the maximum shear stress, recorded in a generic point of 160 structure, which occurs when at least one finite element reaches the yielding stress 161 prescribed by the material model used for the simulations $\left(\sigma_{\mathrm{y}} \approx 46 \mathrm{MPa}\right.$, which is the yield 162 point observed experimentally for antler bone in Gupta et al. $^{20}$ ). The damage initiation 163 values $t_{n}{ }^{0}$ and $t_{s}{ }^{0}$ were chosen such that mode I interfaces are never damageable, while 164 the mode II interfaces could be either damageable or not. Therefore, we adopted a 165 maximum stress criterion for the onset of damage (Equation 2) where damage initiates 166 when the maximum ratio between the traction values at the interface $\left(\left\langle t_{n}\right\rangle, t_{s}\right)$ and the 167 peak values $\left(t_{n}{ }^{0}\right.$ and $\left.t_{s}^{0}\right)$ reaches the value of one. The symbols <> represent the Macaulay 168 brackets that are used to mean that a compressive traction does not initiate the damage.

$$
\max \left\{\frac{\left\langle t_{n}\right\rangle}{t_{n}^{0}}, \frac{t_{s}}{t_{s}^{0}}\right\}=1
$$


171 We used, the values $\mathrm{K}_{\mathrm{nn}}=\mathrm{K}_{\mathrm{ss}}=100 \mathrm{MPa}$ as stiffness coefficients for both mode $\mathrm{I}$ and

172 mode II interfaces. The choice of $K_{n n}$ for the mode II interface and $K_{s s}$ for the mode I

173 interface has no effect on the results. The response of the system was then expected to be 174 mainly affected by $\mathrm{K}_{\mathrm{nn}}$ for the mode I interface and by $\mathrm{K}_{\mathrm{ss}}$ for the mode II one. We 175 performed parametric simulations, keeping all the parameters fixed except for $\mathrm{K}_{\mathrm{nn}}$ for the 176 mode I interface and $\mathrm{K}_{\mathrm{ss}}$ for the mode II interface. We varied these values between 100 177 and $300 \mathrm{MPa} / \mu \mathrm{m}$, with a step of $100 \mathrm{MPa} / \mu \mathrm{m}$ (in total 9 simulations), and we found that 178 when $\mathrm{K}_{\mathrm{nn}}=\mathrm{K}_{\mathrm{ss}}=100 \mathrm{MPa} / \mu \mathrm{m}$ the numerical maximum tissue strain matches the 179 correspondent experimental value closely. We chose these coefficients such that both the 180 numerical and the experimental systems achieve the same level of maximum tissue strain. 181 We expressed the coefficients $K_{n n}$ and $K_{s s}$ as $K$ in Figure $\mathbf{2 b}$, as the figure is 182 representative of a generic mode of fracture. The choice of $\mathrm{K}_{\mathrm{nn}}$ for the mode I interface and $183 \mathrm{~K}_{\mathrm{ss}}$ for the mode II interface is fundamental for the obtained results; in fact it affects not 184 only the deformation of the system but also the hysteretic width of loops in stress-strain 185 curves.

187 As displacements at failure of the interfaces, we adopted the arbitrary high values $10 \mu \mathrm{m}$ 188 and $3 \mu \mathrm{m}$, respectively for the undamageable interfaces and for the damageable interface. 189 These levels of displacement values are never achieved by the finite elements in our 190 simulations, over the course of the stress and strain range seen experimentally, and are 191 hence selected to make sure that the damage occurs only in terms of stiffness degradation 192 and never of complete failure. Specifically, we assumed that the stresses are always 193 transferred through the interfaces. For damageable interfaces, once the damage initiates, 194 the stiffness follows the degradation law: $K^{\prime}=(1-d) K$, where $d \epsilon[0,1]$ is the damageable 195 variable. The total dissipated energy dissipated (per unit of area) through the process of 196 damage of the interface is the area under the traction-separation curve (represented as $\mathrm{G}_{c}$ 
1

2

3

4

5

6

7

8

9

10

11

12

13

14

15

16

17

18

19

20

21

22

23

24

25

26

27

28

29

30

31

32

33

34

35

36

37

38

39

40

41

42

43

44

45

46

47

48

49

50

51

52

53

54

55

56

57

58

59

60
197 in Figure 2b). In Table 1 we schematically summarise the values adopted for the cohesive 198 simulations. 


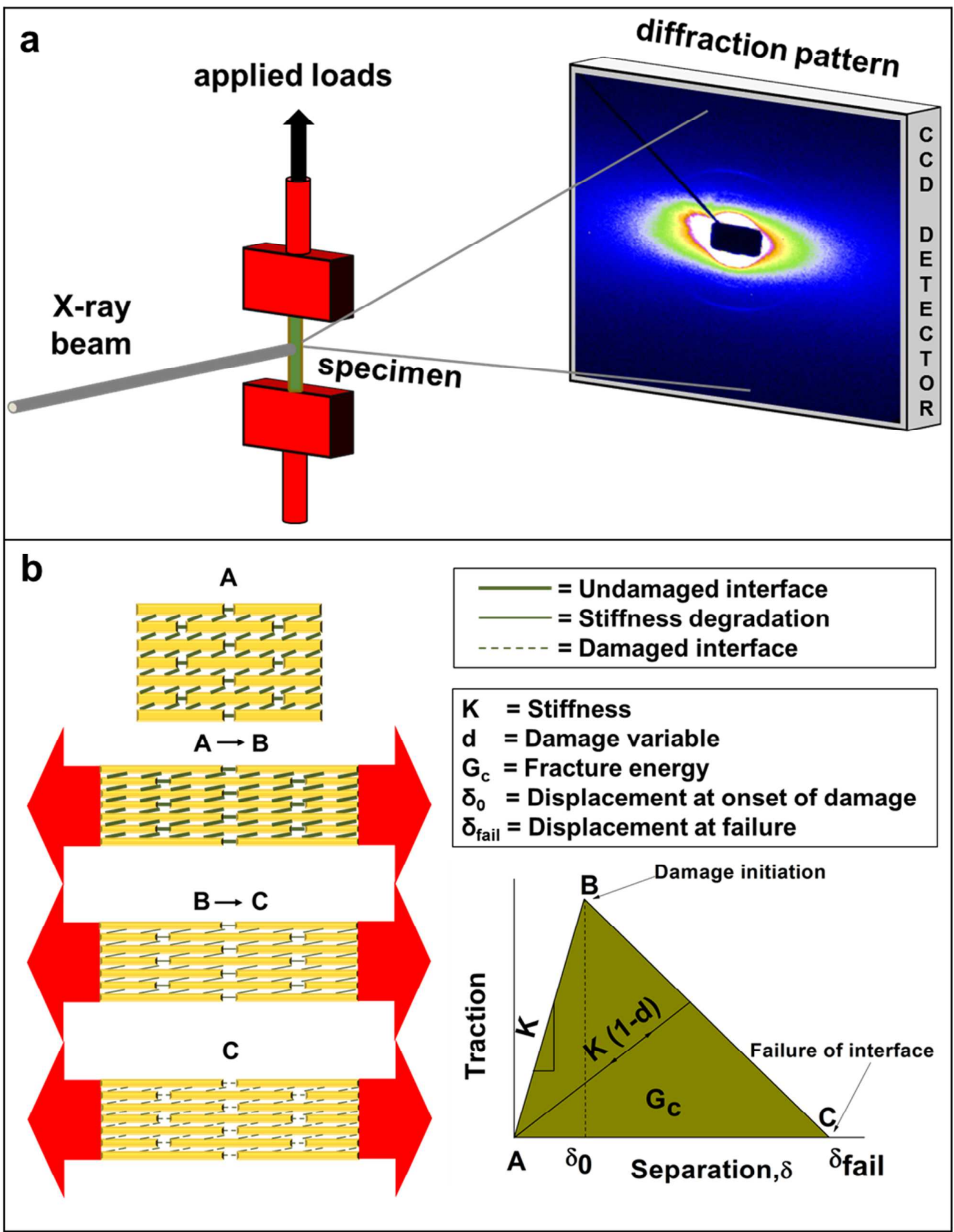

200 Figure 2 - Experimental and numerical methods: (a) experimental setup in synchrotron.

201 Mechanical tester design for tensile testing. (b) Staggered fibril structure at the scale of 0.1 $202-10 \mu m$ with cohesive surface behaviors at the interface. The interfibrillar interface is 
203 modeled with a cohesive-traction separation law. A-B: linear stiffness; B: onset of damage;

204 B-C: damage evolution curve characterized by the stiffness degradation coefficient (1-d) K;

205 C: failure of interface.

206

207

\begin{tabular}{|c|c|c|c|}
\hline & $\begin{array}{c}\text { Mode I } \\
\text { interface }\end{array}$ & $\begin{array}{c}\text { Mode II } \\
\text { undamageable } \\
\text { interface }\end{array}$ & $\begin{array}{c}\text { Mode II } \\
\text { damageable } \\
\text { interface }\end{array}$ \\
\hline \multicolumn{4}{|l|}{ Elastic constant for fracture mode I: } \\
\hline & 100 & 100 & 100 \\
\hline \multicolumn{4}{|l|}{$\mathrm{K}_{\mathrm{nn}}[\mathrm{MPa} / \mu \mathrm{m}]$} \\
\hline \multicolumn{4}{|l|}{ Elastic constant for fracture mode II: } \\
\hline & 100 & 100 & 100 \\
\hline \multicolumn{4}{|l|}{$\mathrm{K}_{\mathrm{ss}}[\mathrm{MPa} / \mu \mathrm{m}]$} \\
\hline \multicolumn{4}{|l|}{ Peak traction for fracture mode I: } \\
\hline & 80 & 80 & 100 \\
\hline
\end{tabular}

Peak traction for fracture mode II:

80

80

0.8

$\mathbf{t}_{\mathrm{s}}{ }^{0}[\mathrm{MPa}]$

Separation value

10

10

3

\section{$\delta[\mu \mathrm{m}]$} the parameters that critically affect the simulations. 
211 The material properties adopted for the fibrils follow a previous model (Figure $3 \mathbf{a})^{20}$, with 212 the Young's modulus of $15.8 \mathrm{GPa}$ and the yielding of $46 \mathrm{MPa}$ (Figure $3 \mathbf{b}$ ). In other words, 213 the elastoplastic behaviour of a single fibril, and its yield point and stiffness, are taken as a 214 given, and arise from previous experimental work (Gupta et al. ${ }^{20,}{ }^{39}$ ). Tensile and cyclic 215 static simulations were performed to study respectively the biphasic fibrillar deformation 216 (section 3.1) and hysteretic loops in stress-strain curves. The applied loads reproduce the 217 values used for experiments by Gupta et al. ${ }^{20}$. Uniaxial traction, along the direction 1 (see 218 Figure 3c), was applied to the top end of the finite element models and fixed support to 219 the bottom end, while right and left sides were kept unconstrained. In detail, a traction 220 value of $60 \mathrm{MPa}$ was imposed for the static tests and a sequence of different traction 221 values for the cyclic tests (43-0 - 50-0 - 56-0-60-0-43 MPa). The uniaxial tissue 222 strain was computed as ratio between the displacement of the loaded edge and the initial 223 length, not by averaging the strain field. This is because the average of the strains over the 224 structure does not account for the deformation of the cohesive interfaces. In fact, since 225 cohesive interfaces are essentially springs, small gaps appear between the fibrils (visible, 226 for example, in Figure 4c). These gaps are not cracks, but only representative of relative 227 displacements between fibrils. 
228

229

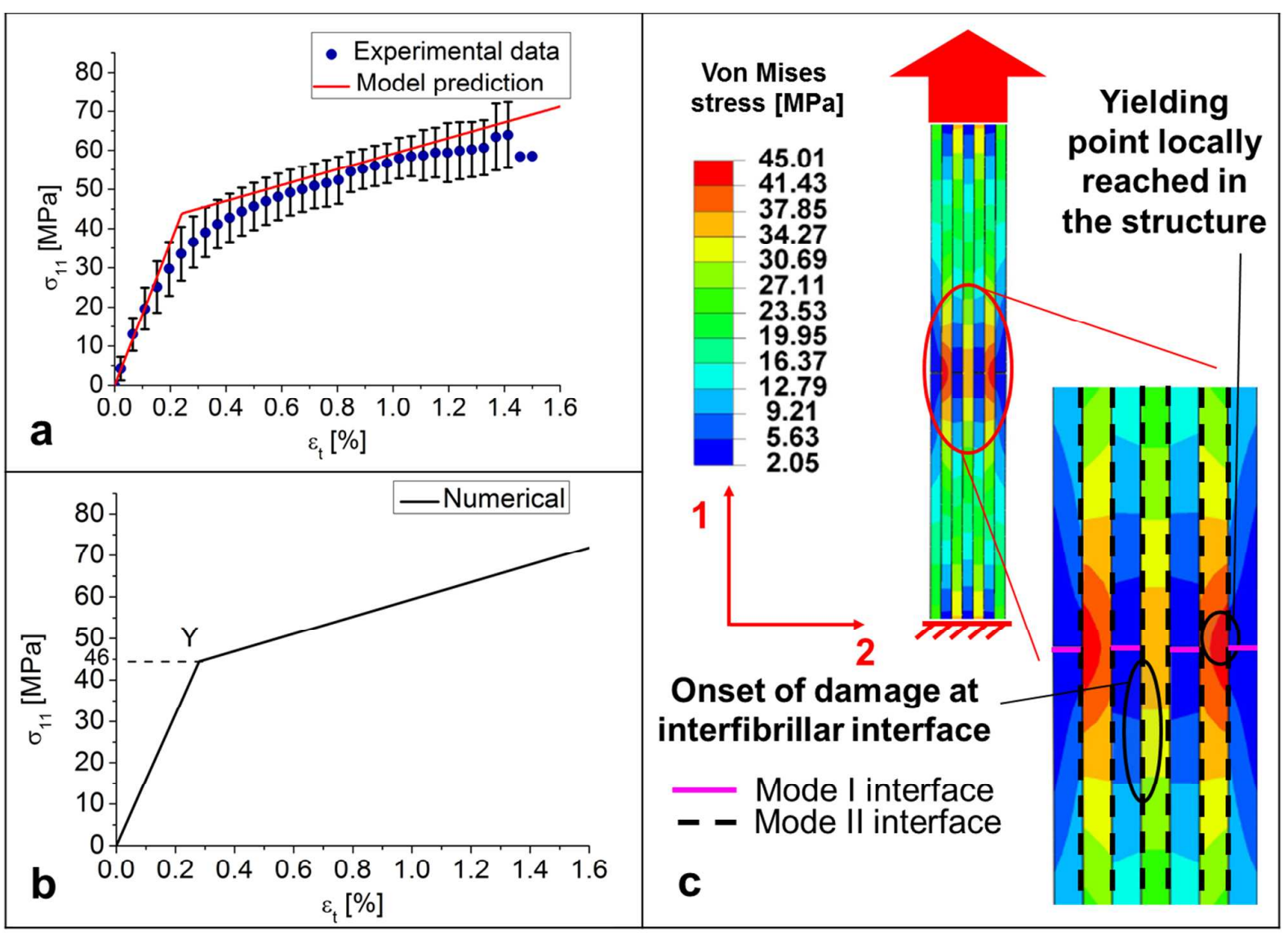

230 Figure 3 - Experimental and numerical fibril stress-strain curves: (a) Experimental

231 stress-strain curve for antler bone versus model prediction. Data are averaged from 10 232 uniaxial stretch-to-failure tests and bars are standard deviations. (b) Material properties 233 input for simulations. (c) Cohesive interface characterization. The interface surrounding the 234 central fibril starts damaging when at least one finite element of the whole structure 235 reaches yielding at $46 \mathrm{MPa}$ (maximum stress criterion).

236

237

238

\section{RESULTS}

239 


\subsection{Biphasic fibrillar deformations}

241 As stated earlier, a main experimental finding in Gupta et al. ${ }^{20}$ was a biphasic fibrillar 242 deformation. Our aim was to understand the role of cohesive interfibrillar surface interfaces 243 in staggered mineralized fibril models, in enabling this behaviour. In this regard the multi244 panel Figure 4 shows an overview comparing strain distributions between experimental 245 data and numerical simulations. These will be explored in detail below.

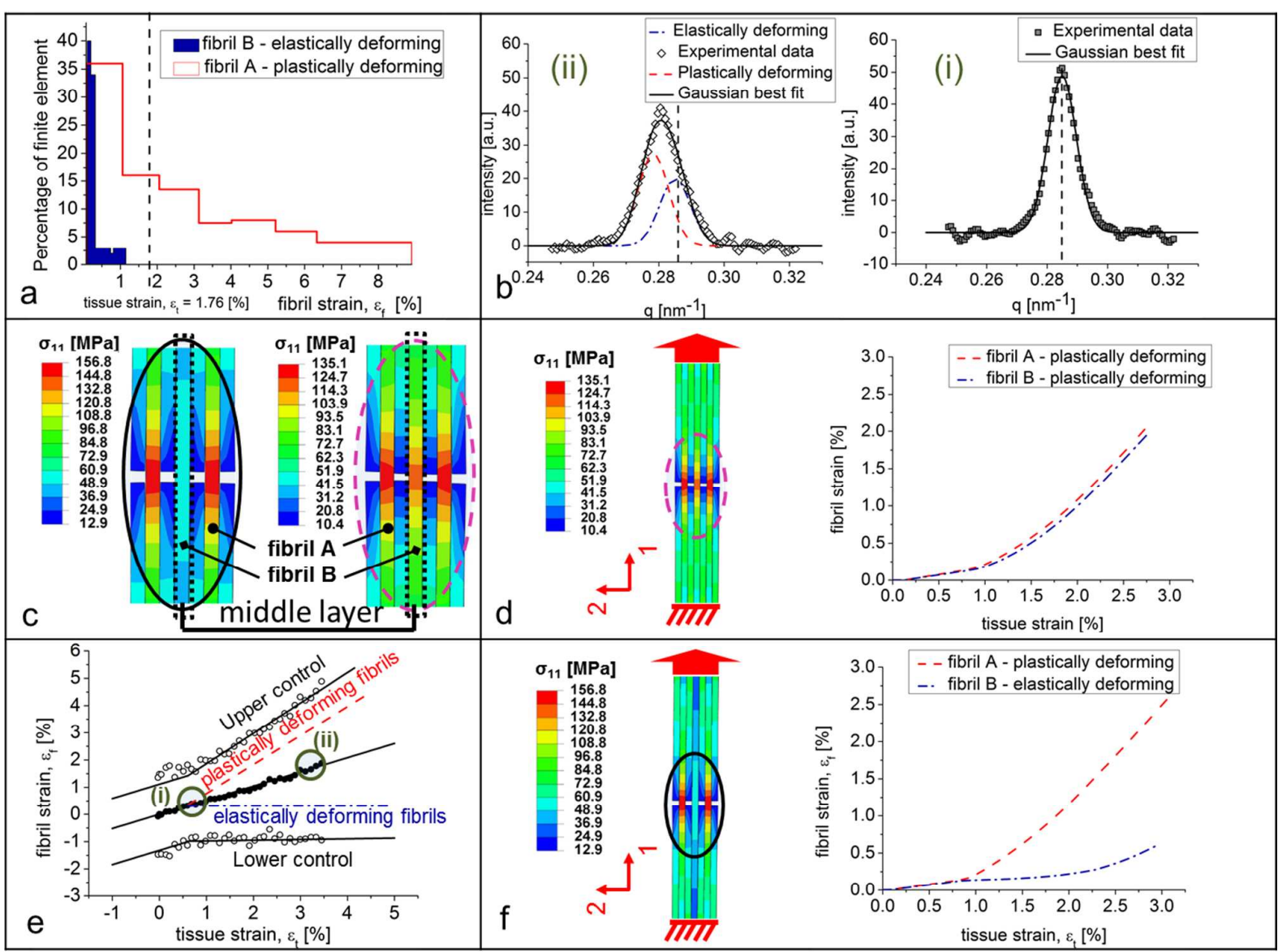

247 Figure 4 - Experimental and numerical fibrillar deformation mechanisms: (a) Strain

248 distribution for elastically and plastically deforming fibrils for a tissue strain value of $1.76 \%$.

249 (b) SAXD intensity plots. (i) Tissue strain at the yield value of $0.6 \%$ (highlighted by a circle 250 and the letter (i) in Figure c). (ii) Tissue strain at the value of $3.2 \%$ (highlighted by a circle 251 and the letter (ii) in Figure c). (c) Stress distributions with and without damageable 252 interfaces. Zoom from Figures $d$ and f. (d) Fibril behaviors in presence of undamageable 
253 interfaces between fibrils. Fibril $b$, at the center of the middle layer, deforms plastically. (e)

254 Variation of mean fibril strain (filled circles) and upper and lower control lines plotted 255 against tissue strain. The middle solid line is the linear regression against tissue strain. (f) 256 Fibril behaviors in presence of damageable interface only between the middle layer and 257 the adjacent two layers. Fibril $b$, at the center of the middle layer, deforms elastically.

258

259

260 For tensile simulations, we firstly adopted a non-damageable law for both the mode I and 261 the mode II interfaces, shown previously in Figure 3c. The results of applied uniaxial 262 traction on the fibrils are shown in Figure 4d, with relative magnification in Figure 4c, with 263 both fibrils A and B plastically deforming, as expected. The maximum longitudinal stress $264\left(\sigma_{11}\right)$, reached in central region of the fibrils, is $135 \mathrm{MPa}$ in fibril A and $119 \mathrm{MPa}$ in fibril $\mathrm{B}$, 265 while the averaged stresses are respectively $65 \mathrm{MPa}$ and $63 \mathrm{MPa}$, beyond the yield point.

267 Secondly, we found that the introduction of damageable mode II interface around the 268 middle layer produces a differentiation of the fibril behaviors. The damage of the interface 269 around the middle layer partially 'isolates' fibril B, which is then not able to fully contribute 270 to the load absorption. While fibril A remains elastoplastic in its deformation behaviour, the 271 deformation of fibril B never exceeds the elastic range (Figures $4 \mathbf{c}-\mathbf{f}$ ). In fact, although 272 the maximum longitudinal stress $\left(\sigma_{11}\right)$, locally measured in a restricted region of fibril $B$, is $27358 \mathrm{MPa}$ (beyond the yield point), its homogenized stress is below the yield point (39 MPa). 274 The corresponding values for fibril A are respectively $157 \mathrm{MPa}$ and $73 \mathrm{MPa}$. Considering a 275 particular level of macroscopic tissue strain beyond the yield point, such as $\varepsilon_{\mathrm{t}}=1.76 \%$ 276 (Figure 4a), we find that most of the load (86 \%) is carried by fibril A whose finite elements 277 are able to stretch up to eight times more than the finite elements in fibril B. For this tissue 278 strain value, the largest deformation in fibril $A$ is $\varepsilon_{f}=8.9 \%$ whilst in fibril $B$ is $\varepsilon_{f}=1.2 \%$. In 
279 addition, the average deformation of fibril $A$ is $0.9 \%$ while for the fibril $B$ the equivalent 280 strain is $0.2 \%$ (below the yield point).

281

282 In Figure 4f, curves show that numerical results are in good agreement with the 283 experimental results shown in Figure 4e, where a comparison between experimental 284 results and model predictions, developed by Gupta et al. ${ }^{20}$, is presented. In particular, the 285 figure includes the upper and lower control lines $\left(\varepsilon_{f, \pm 25 \%}\right)$, the best fit linear regression 286 against tissue strain (middle solid line), and the model predictions for both the elastically 287 deforming fibril (blue dash-dotted line) and the plastically deforming fibrils (red dashed 288 line). In Figures $\mathbf{4 f}-\mathbf{e}$, the elastic and plastic patterns are clearly observable, as also 289 demonstrated by the experimental results in Figure 4b, where the SAXD intensity plot 290 (Figure 4b (i)) shows that all the fibrils are elastic at the yielding point (tissue strain $=0.6$ $291 \%$ ), while for a tissue strain of $3.2 \%$ (Figure $\mathbf{4 b}$ (ii)) the coexistence of plastic and elastic 292 fibrils occurs, with $58 \%$ of fibrils at $\varepsilon_{f}=2.95 \%$ (plastic strain) whilst the remainder fraction 293 at $\varepsilon_{f}=0.53 \%$ (elastic strain). 
$295 \quad 3.2$ The role of mode II interfaces in cyclic loading

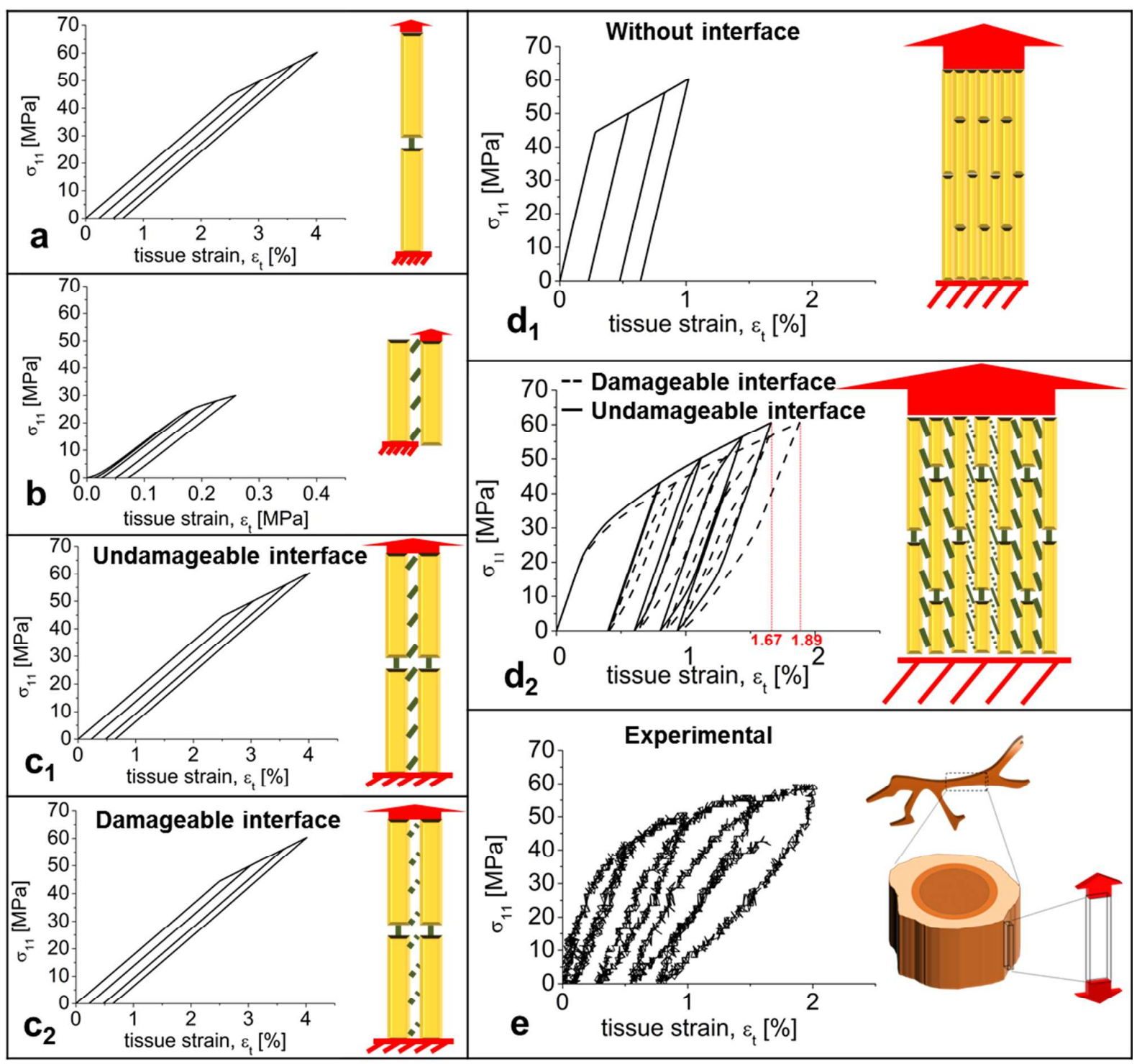

297 Figure 5 - Cyclic loading in different fibrillar geometries: Comparisons between

298 numerical (figures $a, b, c_{1}, c_{2}, d_{1}, d_{2}$ ) and experimental results (figure e). (a) Two fibrils

299 model with 'mode I' non damageable interface. (b) Two fibrils model with 'mode II' non

300 damageable interface. $\left(c_{1}\right)$ Four fibrils model with 'mode l' and 'mode II' non damageable

301 interfaces, in aligned configuration. $\left(c_{2}\right)$ Four fibrils model with 'mode I' non damageable

302 interface and 'mode II' damageable interface, in aligned configuration. ( $\left.d_{1}\right)$ Seven layers

303 model without interfaces, in staggered configuration. $\left(d_{2}\right)$ Comparison between two seven 
304 layers models in staggered configuration: damageable mode II interfaces at the middle

305 layer versus undamageable ones. (e) Experimental results for cyclic loading on bone.

306

307 A second main finding in Gupta et al. ${ }^{20}$ was the existence of hysteresis at the fibrillar level.

308 The elasto-plastic behaviors of a set of different models under cyclic loading were 309 simulated to discover the combined effects of fibril lateral arrangement and architecture, 310 coupled with the interface types described in the previous subsection. We found that the 311 experimentally observed hysteresis in the cyclic loading curves occurs when staggered 312 fibril arrangement coupled with mode I and mode II cohesive surface interfaces are 313 introduced in cyclic simulations. In fact, the presence of only mode I or mode II interfaces 314 for, respectively, a system of two aligned or two-column fibrils is clearly not responsible for 315 hysteresis (Figures $\mathbf{5 a}-\mathbf{b}$ ). These effects do not arise from the limited number of fibrils 316 considered: an increase in number of fibrils from two to four, in a condition of non-overlap, 317 results in no hysteresis with both damageable and undamageable mode II interfaces 318 (Figure 5c1 - c2). As fibrils do not transmit load through shearing in the configurations 319 shown in Figures $\mathbf{5} \mathbf{c} \mathbf{1}-\mathbf{c}_{\mathbf{2}}$, no difference between damageable and undamageable mode 320 II interface is observed. From our set of simulations, we observed that only the 321 concurrence of staggered fibril arrangement and cohesive surface interface (not necessary 322 damageable) leads to hysteresis in loading/unloading stress strain curves (Figure 5d2). It 323 can be seen from Figure 5d1 that staggered but perfectly bonded fibrils (no cohesive 324 interface) do not exhibit hysteresis and loading/unloading patterns perfectly overlap. The 325 introduction of damageable mode II interfaces makes the structure more deformable. 326 Indeed, as fibrils, in staggered configuration, transmit loading through shearing, 327 damageable and then weaker mode II interfaces allow the whole structure to deform up to $3281.89 \%$, while in a condition of non-damageable interfaces the tissue strain reaches the 329 value of $1.67 \%$ (Figure $5 \mathbf{d} 2$ ) at the same stress level of $60 \mathrm{MPa}$. 
331 Numerical results for the cyclic loading curves are in very good agreement with 332 experimental data (comparisons in Figures $\mathbf{5 d 2}$ and $\mathbf{5 e}$ ). Maximum tissue strains, in both 333 cases, are about $1.9 \%$ and furthermore, the structural yielding points occur at tissue strain $334 \approx 0.22 \%$ and axial stress $\approx 25 \mathrm{MPa}$, earlier than the corresponding prediction in the 335 material law ${ }^{20}$ used for simulations.

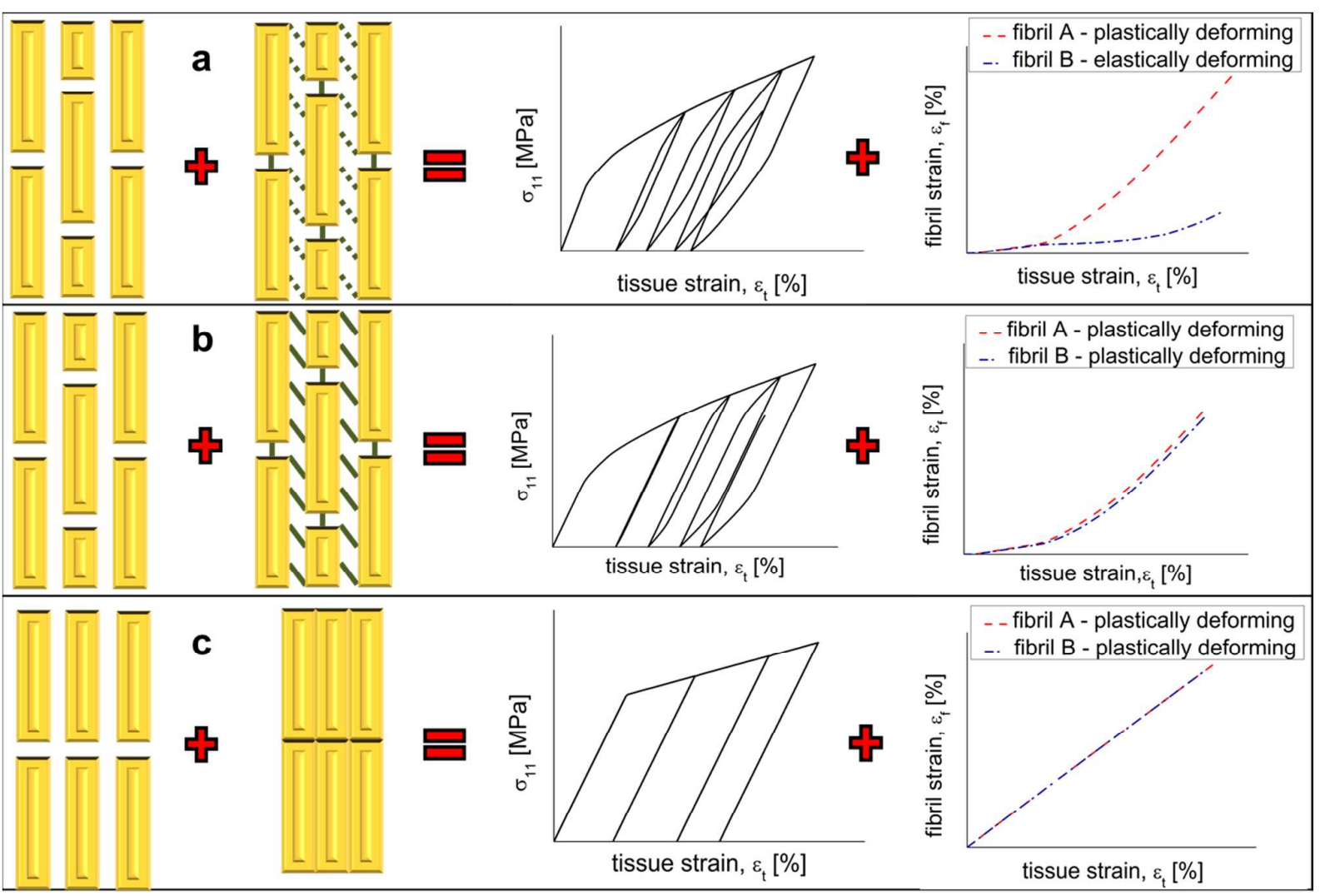

337 Figure 6 - Summary of main results: (a) Staggered fibrillar configuration and 338 damageable mode II interface are responsible for hysteretic stress strain curve and 339 heterogeneous fibrillar deformation. (b) The inclusion of staggered configuration and 340 undamageable interface leads to hysteresis and homogeneous fibril deformation. (c) 341 Aligned fibrillar configuration and no interface lead to no hysteresis and homogeneous fibril 342 deformation. 
344 This paper shows how combination of finite elements simulations at fibrillar level,

345 combined with experimentally derived information on ultrastructural plasticity of the fibril,

346 enables the development of a model for the mechanical behaviors of antler bone under

347 cyclic loading conditions which can explain both the energy dissipation (via hysteresis) as

348 well as the concurrent heterogeneous pattern at the nanoscale. In addition, as shown in

349 Figure 6, our parametric simulations allow us to conclude that the combination of:

a. staggered fibrillar configuration and damageable mode II interface leads to hysteresis and fibrillar heterogeneity;

b. staggered fibrillar configuration and undamageable mode II interface leads to hysteresis and fibrillar homogeneity;

c. aligned fibrillar configuration and perfectly bonded boundary conditions (without cohesive behaviors) at the interfaces leads to no hysteresis and fibrillar homogeneity.

357

\section{DISCUSSION AND CONCLUSION}

Elastic deformation in bone at the nanoscale has been extensively studied ${ }^{4,32,40-42}$ via

360 multiscale fibre composite models that often treat bone material as a two-scale hierarchical

361 composite; mineralized fibrils are arranged in a staggered manner, and fibrils themselves

362 consist of mineral platelets staggered in a collagen matrix phase. Such models are usually

363 validated by comparing the tissue-level modulus predictions to experimentally determined

364 stiffness, though in an ideal scenario predictions of deformation and stress at multiple

365 levels would be calculated and compared to experiment.

366

367 In the area of structural models for inelastic and damage accumulation in bone, there are 368 no modeling attempts for the structural response of the nanoscale bone material under 
369 cyclic loading. To fill this gap, here we proposed a model based on surface cohesive 370 behaviors. Our main assumptions are to neglect the material properties of the interfibrillar 371 matrix and to consider the fibrils linked by cohesive surfaces whose damage process 372 occurs in terms of stiffness degradation. Previously, cohesive behaviours were used for 373 studying the damage mechanisms of bone at different scales ${ }^{43-47}$. Our approach, on the 374 contrary, is based on cohesive stiffness representative of interfaces with negligible small 375 thicknesses. The main difference between the two approaches is that in surface-based 376 laws the damage evolution describes the degradation of the cohesive stiffness whereas in 377 the continuum-based approach ${ }^{43-47}$ the damage concerns the degradation of the material 378 stiffness. The continuum model, called also cohesive zone model, can be used to analyse

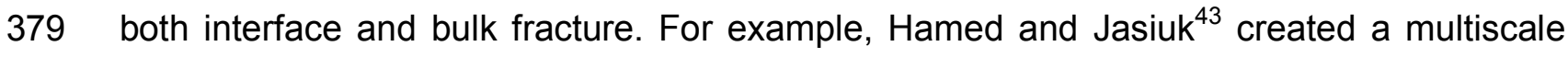
380 model for studying the mechanisms of damage in bone and used the cohesive zone for 381 modelling the fracture of the interface between collagen and hydroxyapatite platelets but 382 also the fracture inside the fibrillar components. They found that the mesh size of cohesive 383 elements had a significant effect on the strength of the mineralized fibrils. A recent study ${ }^{46}$ 384 investigated the evolution of damage in staggered array of mineralised collagen fibrils 385 (MCF) embedded in extrafibrillar protein matrix modelled by continuum cohesive finite 386 elements. The authors found that the failure mechanisms of the extrafibrillar matrix play a 387 dominant role on the energy dissipation capacity of the system. Lin et al. ${ }^{47}$ recently 388 provided evidence as to the importance of the extrafibrillar matrix, considered as 389 composite of hydroxyapatite crystals embedded in an interface modelled by cohesive finite 390 elements, in the pre-yield deformation and failure mode of bone. They found that a tough 391 interface provokes ductile deformation of matrix, as in the case of wet bone, whereas a 392 brittle interface causes brittle deformation, as in dry bone. 
395 Hysteresis, at higher scales in bone, has been found in experiments, but relatively few

396 bone-specific models exist. Ascenzi et al. ${ }^{48}$ tested single osteons and found hysteresis

397 loops under tension. They discovered that the collagen orientation is the main factor to 398 determine the features of hysteresis loops. In both our experimental work and in other 399 references such as Ascenzi et al. ${ }^{48}$, the width of hysteretic loops tends to increase as the 400 applied stress increases. In terms of modelling of the hysteresis loop width, the work of A. 401 G. Evans and co-workers, who carried out modelling and numerical analysis of ceramic 402 matrix composite deformation ${ }^{49}$, is relevant, although their model is applied to a different 403 class of synthetic materials. They derived expressions for which the maximum hysteresis 404 loop width depends on the Young's modulus of both, the fibrils and the matrix, the fibril 405 radius and the fibril volume fraction, but also on the stress conditions, such as the 406 maximum stress reached in the system. At lower scales an analytical model explained the 407 inelastic response of bone ${ }^{50}$, indicating stress/strain hysteresis in loading/unloading tests. 408 The authors found the shear yielding of the interface between collagen fibrils and mineral 409 platelets to be the cause of irreversible slip and then of hysteresis. Hysteresis, in our 410 staggered fibrillar models, is due to the presence of cohesive surface interfaces and in 411 detail, to zones of stress concentration. In fact, we found that, once stretched or released 412 (null surface traction applied to the structure) finite elements of the horizontally adjacent 413 fibrils (fibrils on the same layer) are under-loaded, while finite elements of the underlying 414 fibrils are over-loaded (Figure 4c). This mechanism results in a certain delay of the 415 structure, manifesting as hysteretic loops, necessary to reach the traction value imposed 416 at following loading or unloading steps.

418 Our second finding regards heterogeneous fibrillar deformation caused by partially 419 damageable interfaces. Heterogeneity in the behaviors of mineralized tissue was detected 420 as a mechanism contributing to energy dissipation 19, 22. Mechanical properties of 
421 individual mineralized collagen have been only recently analyzed thanks to the 422 development of innovative experimental techniques. While the fibrillar structure of 423 collagenous tissues was explored extensively during the last decades (for a review see ${ }^{39}$ ), 424 only more recently have their mechanical properties been determined experimentally ${ }^{18,51}$. 425 Heterogeneity in fibrillar deformation were found in antler cortical tissue by Krauss et al. ${ }^{34}$ 426 by using a time-resolved synchrotron small angle $\mathrm{X}$-ray diffraction technique, coupled with 427 tensile testing. They found that heterogeneity in fibrillar deformations starts after the 428 macroscopic yielding. Hang and Barber ${ }^{18}$ performed tensile testing on individual fibrils from 429 antler using atomic force microscopy and scanning electron microscopy. They found 430 heterogeneous deformations in fibrils showing either yield or strain hardening. The 431 structural mechanism for interfacial failure between fibrils may involve the breakage of 432 sacrificial bonds in the noncollageneous proteins found in the interfibrillar matrix ${ }^{22}$. These 433 weak calcium mediated bonds within and between proteins such as osteopontin or 434 osteonectin have been proposed to play a significant role in bone toughness ${ }^{52-53}$ Recently, 435 Poundarik et al. ${ }^{21}$ proposed a mechanism of clusters of extrafibrillar mineral held together 436 by non-collagenous protein glue. Under deformation, this model generates an 437 inhomogeneous strain and stress pattern at the fibrillar level. While we found, instead, that 438 damageable mode II interfaces are responsible for heterogeneous strain fields, the 439 structural origin of such damage may involve mechanisms as proposed by Poundarik et 440 al. $^{21}$. In detail, fibrils surrounded by damageable interfaces behave elastically while other 441 fibrils reach higher stress values after yielding (Figure 7a in Appendix 1). At lower scales, 442 Buehler $^{25}$ found the existence of a range of adhesive energy values between molecule $443\left(0.01-1 \mathrm{~J} / \mathrm{m}^{2}\right)$ which optimizes the toughening mechanisms. Following this concept of 444 optimization, a possible application of our model may be a quantitative and parametric 445 approach to evaluate the types of mechanical behaviors of interfaces which lead to 446 optimized toughness. It must be remarked that in our models, we consider damage only as 
447 stiffness degradation of cohesive surfaces and not as failure and that we use a 2D model.

448 In terms of multiscale modelling of toughness, the current model will allow one to 449 homogenize the properties at the fibrillar scale and these homogenized properties could 450 be inserted into a larger-scale 3D model for the study of crack propagation and damage in 451 real antler bone.

452

453 In conclusion, by constructing a finite element model for the inelastic cyclic loading 454 response of mineralized collagen fibrils in antler, we show that the hysteresis observed is 455 due to interfibrillar staggering leading to inhomogeneous stress fields along the fibril and 456 localized intrafibrillar plasticity, while the inhomogeneous deformation arises from the weak 457 interfaces between fibrils, potentially mediated by sacrificial bonds in the noncollageneous 458 proteins between fibrils.

459

460

\section{ACKNOWLEDGMENTS}

461 HSG thanks Peter Fratzl and Stefanie Krauss (Max Planck Institute of Colloids and 462 Interfaces, Potsdam, Germany), Dr. Sergio Funari (HASYLAB-DESY, beamline A2) and 463 Prof. John Currey (University of York) for very pleasant scientific collaboration on earlier 464 work on antler bone ${ }^{20,34}$. EB is supported by the Queen Mary University of London Start465 up grant for new academics. NMP is supported by the European Research Council (ERC 466 StG Ideas 2011 BIHSNAM n. 279985, ERC PoC 2015 SILKENE nr. 693670), by the 467 European Commission under the Graphene Flagship (WP14 Polymer Nanocomposites, n. 468 696656).

469 470 


\section{REFERENCES}

472 1. Meyers, M. A.; Chen, P.-Y.; Lin, A. Y.-M.; Seki, Y., Biological materials: structure and 473 mechanical properties. Progress in Materials Science 2008, 53 (1), 1-206.

$474 \quad 2 . \quad$ Fratzl, P.; Weinkamer, R., Nature's hierarchical materials. Progress in Materials Science 475 2007, $52(8), 1263-1334$.

$476 \quad 3 . \quad$ Buehler, M. J., Nano-and micromechanical properties of hierarchical biological materials 477 and tissues. Journal of Materials Science 2007, 42 (21), 8765-8770.

478 4. Ji, B.; Gao, H., Mechanical properties of nanostructure of biological materials. Journal of the 479 Mechanics and Physics of Solids 2004, 52 (9), 1963-1990.

$480 \quad 5 . \quad$ Weiner, S.; Wagner, H. D., The material bone: structure-mechanical function relations.

481 Annual Review of Materials Science 1998, 28 (1), 271-298.

482 6. Bonderer, L. J.; Studart, A. R.; Gauckler, L. J., Bioinspired design and assembly of platelet 483 reinforced polymer films. Science 2008, 319 (5866), 1069-1073.

$4847 . \quad$ Pugno, N. M., Mimicking nacre with super-nanotubes for producing optimized super485 composites. Nanotechnology 2006, 17 (21), 5480.

486 8. Bosia, F.; Buehler, M. J.; Pugno, N. M., Hierarchical simulations for the design of 487 supertough nanofibers inspired by spider silk. Physical Review E 2010, 82 (5), 056103.

488 9. Bosia, F.; Abdalrahman, T.; Pugno, N. M., Self-healing of hierarchical materials. Langmuir $4892014,30(4), 1123-1133$.

490 10. Bosia, F.; Abdalrahman, T.; Pugno, N. M., Investigating the role of hierarchy on the strength 491 of composite materials: evidence of a crucial synergy between hierarchy and material mixing.

492 Nanoscale 2012, 4 (4), 1200-1207.

493 11. Wang, Y.; Azaïs, T.; Robin, M.; Vallée, A.; Catania, C.; Legriel, P.; Pehau-Arnaudet, G.;

494 Babonneau, F.; Giraud-Guille, M.-M.; Nassif, N., The predominant role of collagen in the

495 nucleation, growth, structure and orientation of bone apatite. Nature materials 2012, 11 (8), 724496733.

497 12. Dimas, L. S.; Bratzel, G. H.; Eylon, I.; Buehler, M. J., Tough composites inspired by 498 mineralized natural materials: computation, 3D printing, and testing. Advanced Functional 499 Materials 2013, 23 (36), 4629-4638.

500 13. Compton, B. G.; Lewis, J. A., 3D-printing of lightweight cellular composites. Advanced 501 Materials 2014, 26 (34), 5930-5935.

502 14. Koester, K. J.; Ager, J.; Ritchie, R., The true toughness of human cortical bone measured 503 with realistically short cracks. Nature materials 2008, 7 (8), 672-677.

504 15. Fleck, C.; Eifler, D., Deformation behaviour and damage accumulation of cortical bone 505 specimens from the equine tibia under cyclic loading. Journal of biomechanics 2003, 36 (2), 179506189.

507 16. Nalla, R. K.; Kinney, J. H.; Ritchie, R. O., Mechanistic fracture criteria for the failure of 508 human cortical bone. Nature materials 2003, 2 (3), 164-168.

509 17. Vashishth, D.; Behiri, J.; Bonfield, W., Crack growth resistance in cortical bone: concept of 510 microcrack toughening. Journal of biomechanics 1997, 30 (8), 763-769.

511 18. Hang, F.; Barber, A. H., Nano-mechanical properties of individual mineralized collagen 512 fibrils from bone tissue. Journal of the Royal Society Interface 2011, 8 (57), 500-505.

513 19. Tai, K.; Dao, M.; Suresh, S.; Palazoglu, A.; Ortiz, C., Nanoscale heterogeneity promotes 514 energy dissipation in bone. Nature materials 2007, 6 (6), 454-462.

515 20. Gupta, H.; Krauss, S.; Kerschnitzki, M.; Karunaratne, A.; Dunlop, J.; Barber, A.; Boesecke, P.;

516 Funari, S.; Fratzl, P., Intrafibrillar plasticity through mineral/collagen sliding is the dominant 
517 mechanism for the extreme toughness of antler bone. Journal of the mechanical behavior of 518 biomedical materials 2013, 28, 366-382.

519 21. Poundarik, A. A.; Diab, T.; Sroga, G. E.; Ural, A.; Boskey, A. L.; Gundberg, C. M.; Vashishth, 520 D., Dilatational band formation in bone. Proceedings of the National Academy of Sciences 2012, 521109 (47), 19178-19183.

522 22. Fantner, G. E.; Hassenkam, T.; Kindt, J. H.; Weaver, J. C.; Birkedal, H.; Pechenik, L.; Cutroni, 523 J. A.; Cidade, G. A.; Stucky, G. D.; Morse, D. E., Sacrificial bonds and hidden length dissipate energy 524 as mineralized fibrils separate during bone fracture. Nature materials 2005, 4 (8), 612-616.

525 23. Launey, M. E.; Buehler, M. J.; Ritchie, R. O., On the mechanistic origins of toughness in 526 bone. Annual review of materials research 2010, 40, 25-53.

527 24. Tai, K.; Ulm, F.-J.; Ortiz, C., Nanogranular origins of the strength of bone. Nano letters 2006, $5286(11), 2520-2525$.

529 25. Buehler, M. J., Molecular nanomechanics of nascent bone: fibrillar toughening by 530 mineralization. Nanotechnology 2007, 18 (29), 295102.

531 26. Gupta, H. S.; Wagermaier, W.; Zickler, G. A.; Raz-Ben Aroush, D.; Funari, S. S.; Roschger, P.; 532 Wagner, H. D.; Fratzl, P., Nanoscale deformation mechanisms in bone. Nano Letters 2005, 5 (10), 533 2108-2111.

534 27. Zioupos, P.; Currey, J., The extent of microcracking and the morphology of microcracks in 535 damaged bone. Journal of Materials Science 1994, 29 (4), 978-986.

536 28. Schwiedrzik, J.; Raghavan, R.; Bürki, A.; LeNader, V.; Wolfram, U.; Michler, J.; Zysset, P., In 537 situ micropillar compression reveals superior strength and ductility but an absence of damage in 538 lamellar bone. Nature materials 2014, 13 (7), 740-747.

539 29. Barthelat, F.; Rabiei, R., Toughness amplification in natural composites. Journal of the 540 Mechanics and Physics of Solids 2011, 59 (4), 829-840.

541 30. Buehler, M. J.; Yung, Y. C., Deformation and failure of protein materials in physiologically 542 extreme conditions and disease. Nature materials 2009, 8 (3), 175-188.

543 31. Bar-On, B.; Wagner, H. D., Mechanical model for staggered bio-structure. Journal of the 544 Mechanics and Physics of Solids 2011, 59 (9), 1685-1701.

545 32. Bar-On, B.; Wagner, H. D., Structural motifs and elastic properties of hierarchical biological 546 tissues-A review. Journal of structural biology 2013, 183 (2), 149-164.

547 33. Xia, W.; Ruiz, L.; Pugno, N. M.; Keten, S., Critical length scales and strain localization govern 548 the mechanical performance of multi-layer graphene assemblies. Nanoscale 2016, 8 (12), 64565496462.

$550 \quad 34 . \quad$ Krauss, S.; Fratzl, P.; Seto, J.; Currey, J. D.; Estevez, J. A.; Funari, S. S.; Gupta, H. S., 551 Inhomogeneous fibril stretching in antler starts after macroscopic yielding: Indication for a 552 nanoscale toughening mechanism. Bone 2009, 44 (6), 1105-1110.

553 35. Gupta, H. S.; Seto, J.; Wagermaier, W.; Zaslansky, P.; Boesecke, P.; Fratzl, P., Cooperative 554 deformation of mineral and collagen in bone at the nanoscale. Proceedings of the National 555 Academy of Sciences 2006, 103 (47), 17741-17746.

556 36. Dong, X. N.; Almer, J. D.; Wang, X., Post-yield nanomechanics of human cortical bone in 557 compression using synchrotron X-ray scattering techniques. Journal of biomechanics 2011, 44 (4), 558 676-682.

559 37. Zimmermann, E. A.; Schaible, E.; Bale, H.; Barth, H. D.; Tang, S. Y.; Reichert, P.; Busse, B.; 560 Alliston, T.; Ager, J. W.; Ritchie, R. O., Age-related changes in the plasticity and toughness of 561 human cortical bone at multiple length scales. Proceedings of the National Academy of Sciences $562 \quad 2011,108(35), 14416-14421$.

563 38. Xu, X.-P.; Needleman, A., Numerical simulations of fast crack growth in brittle solids. 564 Journal of the Mechanics and Physics of Solids 1994, 42 (9), 1397-1434. 
565 39. Wess, T.; Cairns, D., Nanoarchitectures of the animal extracellular matrix: opportunities for 566 synchrotron radiation studies on collagen and fibrillin. Journal of synchrotron radiation 2005, 12 567 (6), 751-757.

568 40. Hamed, E.; Lee, Y.; Jasiuk, I., Multiscale modeling of elastic properties of cortical bone. Acta 569 Mechanica 2010, $213(1-2), 131-154$.

570 41. Nikolov, S.; Raabe, D., Hierarchical modeling of the elastic properties of bone at submicron 571 scales: the role of extrafibrillar mineralization. Biophysical journal 2008, 94 (11), 4220-4232.

572 42. Jäger, I.; Fratzl, P., Mineralized collagen fibrils: a mechanical model with a staggered 573 arrangement of mineral particles. Biophysical journal 2000, 79 (4), 1737-1746.

574 43. Hamed, E.; Jasiuk, I., Multiscale damage and strength of lamellar bone modeled by 575 cohesive finite elements. journal of the mechanical behavior of biomedical materials 2013, 28, 94576110.

577 44. Ural, A., Prediction of Colles' fracture load in human radius using cohesive finite element 578 modeling. Journal of biomechanics 2009, 42 (1), 22-28.

579 45. Mischinski, S.; Ural, A., Finite element modeling of microcrack growth in cortical bone. 580 Journal of Applied Mechanics 2011, 78 (4), 041016.

581 46. Lai, Z. B.; Yan, C., Mechanical behaviour of staggered array of mineralised collagen fibrils in 582 protein matrix: Effects of fibril dimensions and failure energy in protein matrix. Journal of the 583 Mechanical Behavior of Biomedical Materials 2017, 65, 236-247.

584 47. Lin, L.; Samuel, J.; Zeng, X.; Wang, X., Contribution of extrafibrillar matrix to the mechanical 585 behavior of bone using a novel cohesive finite element model. Journal of the Mechanical Behavior 586 of Biomedical Materials 2017, 65, 224-235.

587 48. Ascenzi, A.; Benvenuti, A.; Mango, F.; Simili, R., Mechanical hysteresis loops from single 588 osteons: technical devices and preliminary results. Journal of biomechanics 1985, 18 (5), 391-398.

589 49. Vagaggini, E.; Domergue, J. M.; Evans, A. G., Relationships between hysteresis 590 measurements and the constituent properties of ceramic matrix composites: I, theory. Journal of 591 the American Ceramic Society 1995, 78 (10), 2709-2720.

592 50. Mercer, C.; He, M.; Wang, R.; Evans, A., Mechanisms governing the inelastic deformation of 593 cortical bone and application to trabecular bone. Acta Biomaterialia 2006, 2 (1), 59-68.

594 51. Shen, Z. L.; Dodge, M. R.; Kahn, H.; Ballarini, R.; Eppell, S. J., Stress-strain experiments on 595 individual collagen fibrils. Biophysical Journal 2008, 95 (8), 3956-3963.

596 52. Thurner, P. J.; Chen, C. G.; Ionova-Martin, S.; Sun, L.; Harman, A.; Porter, A.; Ager, J. W.; 597 Ritchie, R. O.; Alliston, T., Osteopontin deficiency increases bone fragility but preserves bone mass. 598 Bone 2010, 46 (6), 1564-1573.

599 53. Gupta, H. S.; Fratzl, P.; Kerschnitzki, M.; Benecke, G.; Wagermaier, W.; Kirchner, H. O., 600 Evidence for an elementary process in bone plasticity with an activation enthalpy of $1 \mathrm{eV}$. Journal 601 of the Royal Society Interface 2007, 4 (13), 277-282.

602 


\section{For Table of Contents Use Only}

605

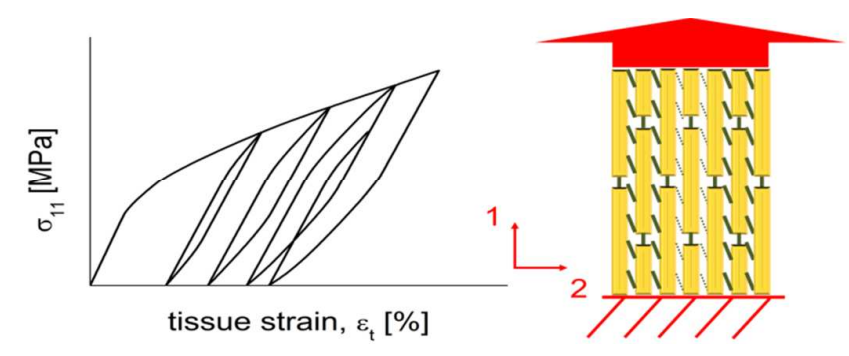

606

607 Staggered fibrils and damageable interfaces lead concurrently and independently to 608 hysteretic energy absorption and inhomogeneous strain fields in cyclically loaded antler 609 bone

610 P. De Falco, E. Barbieri, N. Pugno and H. S. Gupta

611 